\title{
Incarcerated Right Lateral Trocar-Site Hernia as a Rare Complication of Robot-Assisted Laparoscopic Prostatectomy
}

\author{
Neil J. Kocher, MD, Daniel llinsky, BS, and Jay D. Raman, MD, FACS ${ }^{1}$
}

\begin{abstract}
Background: Trocar-site hernia (TSH) is an uncommon complication following laparoscopic surgery and a potential cause of significant morbidity if bowel incarceration or strangulation occurs. Lateral trocar sites are intrinsically less susceptible to hernia development and traditionally do not mandate fascial closure. We present an unusual case involving an incarcerated TSH from a 12-mm right lateral port after robotic prostatectomy.

Case Presentation: The patient is a 76-year-old man with localized intermediate risk, high-volume prostate cancer who underwent a robot-assisted, laparoscopic prostatectomy. He developed generalized malaise and no flatus or bowel movements at 7 days postoperatively. Cross-sectional imaging revealed small bowel dilatation with a transition point along the right lateral 12-mm trocar site. A minilaparotomy (extension of lateral port incision) confirmed incarcerated but viable small bowel. This was reduced and fascia closed without surgical resection.

Conclusion: While rare, TSH is an important clinical entity to recognize after minimally invasive surgery. It is critical to properly review trocar technique and maintain a low threshold for close postoperative follow-up, if there is clinical concern for a trocar hernia.
\end{abstract}

Keywords: incarcerated trocar-site hernia, robotic prostatectomy, prostate cancer, minimally invasive surgery

\section{Introduction and Background}

$\mathbf{T}$ ROCAR-SITE HERNIAS (TSH) are uncommon, but potentially a source of major complications following laparoscopic surgery. The estimated incidence of TSH is low, ranging from $0 \%$ to $5.2 \%$, and the majority of hernias located along the umbilicus. ${ }^{1}$ Lateral trocar sites are intrinsically less susceptible to hernia development due to coaptation of flank musculature traditionally do not mandate fascial closure. In this case report, we present the case of a 76-year-old male who underwent robot-assisted, laparoscopic prostatectomy for intermediate-risk, largevolume, and clinically localized prostate cancer, complicated postoperatively by an incarcerated hernia along the $12-\mathrm{mm}$ right lateral trocar site requiring operative bowel reduction and closure.

\section{Case Presentation}

A 76-year-old Caucasian man with body mass index (BMI) of 32 and diabetes mellitus underwent a complicated robotassisted laparoscopic prostatectomy for Gleason $4=3=7$, pT3bN0Mx prostate cancer with 17 lymph nodes removed. No intraoperative issues were noted with any of the trocar sites. Seven days following the procedure, he presented to the Emergency Department with generalized weakness, decreased oral intake, absence of flatus, obstipation, and no bowel movements for 72 hours. On triage assessment he was hypotensive, tachycardic, and tachypneic, but without appreciable peritoneal signs such as rebound tenderness or abdominal guarding. His initial hypotension and tachycardia improved with 2 L IV fluid resuscitation. Laboratory studies revealed acute kidney injury with elevated creatinine to 3.0

\footnotetext{
${ }^{1}$ Division of Urology, Department of Surgery, College of Medicine, The Pennsylvania State University, Hershey, Pennsylvania.

${ }^{2}$ College of Medicine, The Pennsylvania State University, Hershey, Pennsylvania.
}

(C) Neil J. Kocher et al. 2017; Published by Mary Ann Liebert, Inc. This is an Open Access article distributed under the terms of the Creative Commons Attribution License, which permits unrestricted use, distribution, and reproduction in any medium, provided the original work is properly cited. 
FIG. 1. Noncontrast CT axial and coronal images. Incarcerated small bowel loop within the right lateral trocar site, with a transition point in the right lower quadrant (red circle).

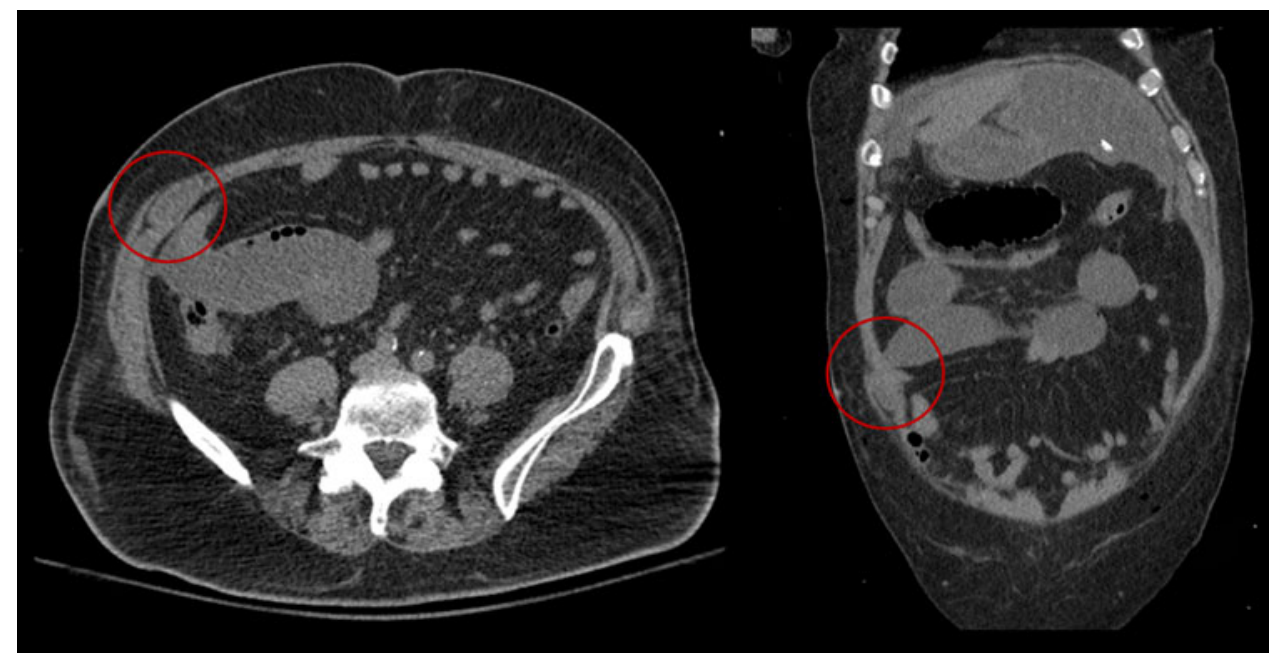

(baseline 0.9), mild elevation of liver function tests, lactic acid level of 12, and a normal hemoglobin level. Noncontrast abdominopelvic CT scan identified a significantly distended stomach as well as small bowel dilatation with a transition point along the right lower quadrant abdominal wall at his 12-mm right lateral trocar site (Fig. 1). On physical examination, there was no palpable bulge or overlying erythema along the healing right lateral incision.

The patient was transported to the operating room and a $4 \mathrm{~cm}$ minilaparotomy was performed over the right lateral incision in conjunction with the general surgery service. On exploration, the incarcerated small bowel loop was identified, visually hyperemic, but otherwise viable. This was easily reduced without resection and the right lateral wall defect was closed primarily. Postoperatively, the patient was diagnosed with a left lower lobe pneumonia based on clinical findings and consolidation on chest $\mathrm{x}$-ray. His bowel function ultimately normalized and he was discharged to home on postlaparotomy day 8 . Patient has recovered well since then with PSA 3 months postprocedure being undetectable.

\section{Discussion}

$\mathrm{TSH}$ is a rare complication following laparoscopic surgery. Limited data demonstrate an incidence between $0.4 \%$ and $0.9 \%$ after robotic prostatectomy. ${ }^{2}$ TSH may be a consequence of technical factors, such as trocar design, port location, or fascial closure, and/or patient-related factors, including age, BMI, or wound infection. ${ }^{3}$ Most laparoscopic surgeons now use bladeless, radiating trocars to decrease complications by penetrating muscle layers via a splitting process rather than cutting. ${ }^{2}$

Closing lateral port site fascia after using bladeless trocars is a controversial topic. Theoretically, the multiple layers of lateral flank musculature should coapt upon removal of trocars. Although some studies advocate that not closing fascia is safe and effective when using bladeless trocars, there have been several reports of incisional hernias after their use. ${ }^{2}$ TSH incidence increases with trocar size, with 12-mm trocars having the greatest risk. ${ }^{1}$ Traditionally fascial closure was only done for $12-\mathrm{mm}$ periumbilical ports as these are the primary sites of trocar hernias after laparoscopic procedures. ${ }^{1}$

Regarding robotic prostatectomy, Kang et al. reviewed 498 patients where only the fascia of the $12-\mathrm{mm}$ umbilical port site was closed and noted that there were no right lateral 12-mm trocar hernias. ${ }^{4}$ Conversely, Chiong et al. retrospectively reviewed 1055 patients who underwent laparoscopic urologic surgery and found that out of the 7 hernia cases reported, 6 were located at the 12-mm lateral port sites, and specifically, all 4 incisional hernias from patients undergoing robot-assisted laparoscopic prostatectomy involved the $12-\mathrm{mm}$ lateral port sites. ${ }^{2}$

While a rare occurrence, the available literature and this patient experience influenced our technique to close all 12-mm trocar sites, regardless of location. Ultimately, in an effort to prevent trocar hernia formation and subsequent complications, it is critical to properly review trocar technique and maintain a low threshold for close postoperative follow-up, if there is clinical concern for a trocar hernia.

\section{Conclusion}

We report a case of an incarcerated hernia from a 12-mm lateral port site in a 76-year-old male after robot-assisted, laparoscopic prostatectomy. TSH is a potential cause of significant morbidity if incarceration or strangulation occurs. Ultimately, in an effort to prevent trocar hernia formation and subsequent complications, it is critical to properly review trocar technique and maintain a low threshold for close postoperative follow-up if there is clinical concern for TSH development.

\section{Acknowledgment}

The authors graciously thank the Keith and Lynda Haring Urologic Research Fund for support of this work.

\section{Disclosure Statement}

No competing financial interests exist.

\section{References}

1. Helgstrand F, Rosenberg J, Bisgaard T. Trocar site hernia after laparoscopic surgery: A qualitative systematic review. Hernia 2011;15:113-121.

2. Chiong E, Hegarty PK, Davis JW, Kamat AM, Pisters LL, Matin SF. Port-site hernias occurring after the use of bladeless radially expanding trocars. Urology 2010;75:574-580.

3. Tonouchi H, Ohmori Y, Kobayashi M, Kusunoki M. Trocar site hernia. Arch Surg 2004;139:1248-1256. 
4. Kang DI, Woo SH, Lee DH, Kim IY. Incidence of port-site hernias after robot-assisted radical prostatectomy with the fascial closure of only the midline $12-\mathrm{mm}$ port site. J Endourol 2012;26:848-851.

Address correspondence to: Jay D. Raman, MD, FACS Division of Urology Department of Surgery College of Medicine

The Pennsylvania State University 500 University Drive, H055

Hershey, PA 17033-0850

E-mail: jraman@pennstatehealth.psu.edu

\section{Abbreviations Used \\ $\mathrm{CT}=$ computed tomography \\ $\mathrm{TSH}=$ trocar-site hernia}

Cite this article as: Kocher NJ, Ilinsky D, Raman JD (2017) Incarcerated right lateral trocar site hernia as a rare complication of robot-assisted laparoscopic prostatectomy, Journal of Endourology Case Reports 3:1, 155-157, DOI: 10.1089/cren.2017.0099. 